\title{
Neurocircuitry underlying stress and emotional regulation in animals prenatally exposed to alcohol and subjected to chronic mild stress in adulthood
}

\section{Charlis Raineki ${ }^{1}$, Kim G. C. Hellemans ${ }^{1,2}$, Tamara Bodnar ${ }^{1}$, Katie M. Lavigne ${ }^{3,4}$, Linda Ellis ${ }^{1}$, Todd S. Woodward ${ }^{3,4}$ and Joanne Weinberg ${ }^{1 *}$}

1 Department of Cellular and Physiological Sciences, The University of British Columbia, Vancouver, BC, Canada

${ }^{2}$ Department of Neuroscience, Carleton University, Ottawa, ON, Canada

${ }^{3}$ Department of Psychiatry, The University of British Columbia, Vancouver, BC, Canada

${ }^{4}$ BC Mental Health and Addictions Research Institute (BCMHARI), Vancouver, BC, Canada

Edited by:

Nikolaos P. Daskalakis, Icahn School of Medicine at Mount Sinai, USA

Reviewed by:

Gábor B. Makara, Hungarian Academy of Sciences, Hungary

Ishwar Parhar, Monash University, Malaysia

\section{*Correspondence:}

Joanne Weinberg, Department of Cellular and Physiological Sciences, The University of British Columbia, 2350 Health Sciences Mall,

Vancouver, BC V6T 1Z3, Canada e-mail: joanne.weinberg@ubc.ca
Individuals exposed to alcohol during gestation show higher rates of psychopathologies. The hyperresponsivity to stress induced by prenatal alcohol exposure (PAE) may be related to this increased rate of psychopathologies, especially because this population is more likely to be exposed to stressful environments throughout life. However, alcohol-induced changes in the overlapping neurocircuitries that underlie stress and the expression of psychopathologies are not fully understood. Here, we performed a comprehensive analysis of the neural activity within central areas known to play key roles in both emotional and stress regulation. Adult male and female offspring from PAE, pairfed, and ad libitum-fed control conditions were exposed to chronic mild stress (CMS). Following CMS, the neural activity (c-fos mRNA) of the amygdala, ventral hippocampal formation, medial prefrontal cortex (mPFC), and paraventricular nucleus of hypothalamus (PVN) was assessed in response to an acute stress (elevated plus maze). Our results demonstrate that, overall, PAE decreased neural activity within the amygdala and hippocampal formation in males and increased neural activity within the amygdala and MPFC in females. CMS reduced neural activity within the $\mathrm{MPFC}$ and PVN in PAE males, but reduced activity in all areas analyzed in control males. By contrast, CMS reduced neural activity in the MPFC in PAE females and had no effects in control females. Furthermore, the constrained principal component analysis revealed that these patterns of neural activity resulted in differential activation of the functional neural networks in males compared to females, indicating sexually dimorphic effects of PAE and CMS. Importantly, the altered networks of brain activation in PAE animals may underlie the hyperresponsivity to stress and increased psychopathologies observed among individuals prenatally exposed to alcohol.

Keywords: prenatal alcohol exposure, chronic mild stress, amygdala, hippocampus, prefrontal cortex, paraventricular nucleus of hypothalamus, $c$-fos, constrained principal component analysis

\section{INTRODUCTION}

Fetal development is a dynamic process strongly influenced by the quality of the environment in which it occurs $(1,2)$. Adverse intrauterine environments can negatively alter the developmental trajectory, resulting in physical and mental health problems later in life $(1,3)$. In mammals, the mother plays a critical role in fetal development, not only by supplying nutrients and oxygen via the placenta, but also by modulating the environmental stimuli to which the fetus is exposed (4). Clinical and experimental studies have clearly demonstrated that alcohol consumed during pregnancy has pervasive and long-lasting negative effects on fetal development (5-9). Indeed, prenatal alcohol exposure (PAE) is strongly associated with a wide range of neural, behavioral, hormonal, and cognitive deficits in humans, non-human primates, and rodents (5-9). In addition, the rates of psychopathologies (e.g., anxiety, depression, other mood disorders, and substance use disorders) among individuals prenatally exposed to alcohol are disproportionately higher when compared to unexposed individuals (1013). Unfortunately, little is known about the underlying neural processes that support this high incidence of psychopathologies following PAE.

Brain areas implicated in psychopathologies overlap to a large extent with areas that mediate responses to stress. The amygdala, hippocampus, and medial prefrontal cortex (mPFC) are intrinsically involved in the modulation of hypothalamic-pituitaryadrenal (HPA) axis activity $(14,15)$, and are also associated with several mental health disorders $(16,17)$. Abnormal function of any one of these highly interconnected areas may result in abnormal responses to stress and/or the emergence of psychopathologies. This is especially relevant for individuals exposed to alcohol during gestation, as neuroimaging studies have demonstrated structural and functional alterations in brain regions 
involved in stress regulation, such as the hippocampus and PFC (18-21).

Not surprisingly, a striking deficit induced by PAE involves changes in how affected individuals process and/or respond to a stressful stimulus or situation. The human and animal literature clearly demonstrates that alcohol consumption during pregnancy results in offspring that are hyperresponsive to a variety of stressors (5, 9,22-24). For example, children exposed to alcohol in utero show higher salivary cortisol levels following exposure to stressors such as blood draw and the still-face procedure when compared to unexposed counterparts $(22,23)$. Additionally, higher basal cortisol levels were also observed in children exposed to alcohol prenatally $(23,24)$. In parallel with these studies, rats prenatally exposed to alcohol show increased HPA activation and/or a delay in return to basal levels as compared to controls in response to a wide range of stressful stimuli including footshock, restraint, immune challenge, and exposure to novel environments (25-30). In addition, HPA dysregulation is observed not only following stress, but also under basal conditions, even in the face of similar basal hormone levels. Dysregulation is evident at multiple levels of the axis, and appears to reflect changes in both HPA drive and feedback regulation and/or in the balance between drive and feedback. Taken together, these findings suggest that for individuals prenatally exposed to alcohol, stressors occurring over the life span may be acting on an already dysregulated or sensitized stress neurocircuitry. Thus, stress hyperresponsivity and HPA dysregulation may be a crucial factor mediating the increased vulnerability of these individuals to develop later psychopathologies $(5,31,32)$. An aggravating factor to this already unfavorable situation is that individuals prenatally exposed to alcohol are, in general, at a higher risk of encountering a more stressful environment throughout the lifespan $(12,13,33)$.

Using a rodent model of PAE, our laboratory has been successful in demonstrating that the combination of PAE and chronic unpredictable stress in adulthood increases anxiety- and depressive-like behaviors in a sexually dimorphic manner $(5,31$, 32). However, the neurocircuitry underlying both stress dysregulation and anxiety- and depressive-like behaviors of individuals prenatally exposed to alcohol is still not fully understood. In the present study, adult male and female rats that were prenatally exposed to alcohol underwent a 10-day chronic mild stress (CMS) regimen in adulthood, which was followed by a comprehensive analysis of the neural activity within brain areas known to play key roles in stress and emotional regulation. Specifically, we assessed $c$-fos mRNA expression as a measurement of neural activity in the medial parvocellular dorsal division of the paraventricular nucleus of hypothalamus (mpdPVN), ventral hippocampal formation (CA1, CA3, DG, and ventral subiculum), the amygdala (central, cortical, lateral, basal, and medial nuclei), and the mPFC [anterior cigulate cortex (ACC), prelimbic (PrL), and infralimbic (IL)] in response to an acute stressor-exposure to the elevated plus maze $(34,35)$. In order to identify networks of coordinated activity in these brain regions rather than simply to identify whether activity differed between experimental groups for each individual brain area, we employed constrained principal component analysis (CPCA) in addition to a traditional univariate analysis (ANOVAs followed by post hoc group comparisons). CPCA is a multivariate technique that combines multivariate multiple regression and principal component analysis (PCA) into a unified framework (36-41). In the current study, CPCA allowed for the identification of brain networks that were specifically related to our experimental conditions (i.e., PAE and CMS). Thus, we were able to identify the networks of brain regions associated with differences in neural activity between animals that were prenatally exposed to alcohol, with or without CMS exposure in adulthood, and their respective controls. Below, we present results from a traditional univariate technique (ANOVA) as well as CPCA to demonstrate how this multivariate technique can facilitate interpretation when group differences are observed in a variety of distinct, but interconnected, brain regions.

\section{MATERIALS AND METHODS}

\section{ANIMALS AND BREEDING}

Female Sprague-Dawley rats were obtained from Charles River Laboratories (St. Constant, QC, Canada) and male SpragueDawley rats were obtained from the UBC Animal Care Centre. Rats were pair-housed by sex and maintained at a constant temperature $\left(21 \pm 1^{\circ} \mathrm{C}\right)$ and on a 12-h light-dark cycle (lights on at 6 a.m.) with ad libitum access to water and standard laboratory chow (Jamieson's Pet Food Distributors Ltd., Canada). After a 10-day acclimation period, male and female pairs were placed together suspended in stainless steel cages with mesh front and floor $(25 \mathrm{~cm} \times 18 \mathrm{~cm} \times 18 \mathrm{~cm})$. Day 1 of gestation $(\mathrm{G} 1)$ was indicated by the presence of a vaginal plug on the wax paper beneath the breeding cages, which were checked daily. All experiments were performed in accordance with National Institutes of Health (NIH) guidelines for the care and use of laboratory animals and the Canadian Council on Animal Care guidelines and were approved by The University of British Columbia Animal Care Committee.

\section{PRENATAL ALCOHOL EXPOSURE}

On G1, females were single-housed and randomly assigned to one of the three prenatal treatment groups: PAE, Pair-Fed (PF), or ad libitum-fed Control (C). Dams in the PAE group were offered ad libitum liquid ethanol diet with 36\% ethanol-derived calories (Dyets, Inc., Bethlehem, PA, USA). This diet is formulated to provide adequate nutrition to pregnant rats regardless of ethanol intake $(42,43)$. PF dams were offered a liquid control diet with maltose-dextrin isocalorically substituted for ethanol, in an amount matched to the consumption of a PAE partner according to gestation day (gram/kilogram body weight/day of gestation). The control dams were offered ad libitum access to standard laboratory chow (Jamieson's Pet Food Distributors Ltd.). All animals were provided with fresh diet daily within $1 \mathrm{~h}$ of lights off to prevent a shift in corticosterone circadian rhythms, which occurs in animals that are on a restricted feeding schedule, such as the PF dams $(44,45)$. Experimental diets were continued through G21. Beginning on G22, all animals were offered ad libitum access to standard laboratory chow and water, which they received throughout lactation. Pregnant dams were left undisturbed except for cage changing and weighing, which occurred on G1, G7, G14, and G21. On the day of birth [postnatal day 1 (PN1)], litters were weighed and culled to 10 pups with an attempt to preserve an equal number of males and females per litter. Dams and pups were weighed 
on PN1, PN8, PN15, and PN22 [dam and pup body weight data were published in Ref. (31)]. On PN22, pups were weaned and group-housed by litter and sex.

\section{CHRONIC MILD STRESS}

On PN40, one male and one female rat from each litter were randomly assigned to either the CMS or non-CMS condition and were pair-housed with another animal of the same sex, prenatal treatment, and stress condition (CMS or non-CMS). In adulthood (PN60-90), CMS animals were subjected to 10 consecutive days of randomized stressors. Animals were exposed to two different stressors each day: the first at a randomized time in the morning (between 8:00 and 12:00 h) and the second at a randomized time in the afternoon (between 13:00 and 16:00 h), with a minimum of $2 \mathrm{~h}$ between stressors. On day 1 of CMS, all animals were weighed, handled, and moved to new colony rooms in a neighboring building. CMS and non-CMS animals were housed in separate colony rooms so that non-CMS animals were not exposed to the disturbance of either moving CMS animals between stressors, or stress odors and vocalizations produced by CMS animals. CMS exposure occurred in a room separate from the colony room. The order and type of stressor were randomized, but all animals received the same number of exposures to each stressor over the 10-day period. Stressors included: (1) platform: $5 \mathrm{~min}$ exposure to an elevated Plexiglass platform $(20 \mathrm{~cm} \times 20 \mathrm{~cm})$ mounted on a 90 -cm high post; (2) cage tilt: the home cage was tilted at a $30^{\circ}$ angle for $2 \mathrm{~h}$; (3) novel cage: exposure to a novel, small $(18 \mathrm{~cm} \times 25 \mathrm{~cm} \times 15 \mathrm{~cm})$, and opaque cage without food and water for $1 \mathrm{~h}$; (4) soiled cage: exposure to the soiled cage from another sex-matched pair of animals for $1 \mathrm{~h}$; (5) restraint: restraint in PVC tubes $(15 \mathrm{~cm} \times 6 \mathrm{~cm}$ for females and $19 \mathrm{~cm} \times 7 \mathrm{~cm}$ for males) for $30 \mathrm{~min}$; (6) social isolation: overnight isolation in hanging wire mesh cage $(20 \mathrm{~cm} \times 23 \mathrm{~cm} \times 18 \mathrm{~cm})$ without food and water for $12 \mathrm{~h}$; (7) white noise: exposure to white noise ( $40 \mathrm{~dB}$; Lafayette Instruments model no. 15800) for $2 \mathrm{~h}$; and (8) tail nick: a cut was made $1 \mathrm{~mm}$ from the tip of the tail for blood collection from the tail vein [blood sample data was published in Ref. (31)]. Non-CMS animals remained undisturbed other than routine husbandry during this same period.

\section{ACUTE STRESS (ELEVATED PLUS MAZE EXPOSURE) AND BRAIN COLLECTION}

The day after the end of CMS (day 11), all animals from both CMS and non-CMS conditions were habituated to the behavioral testing room for $10 \mathrm{~min}$. The following day (day 12), animals were exposed to an elevated plus maze for $5 \mathrm{~min}$ in a dimly lit room during the light phase (09:00 and 12:00 h) of the lightdark cycle [elevated plus maze data were published in Ref. (31)]. Exposure to the elevated plus maze is a stressor known to produce an increase in corticosterone levels (35). The elevated plus maze consisted of two closed arms $(69 \mathrm{~cm} \times 10.5 \mathrm{~cm})$ and two open arms $(69 \mathrm{~cm} \times 10.5 \mathrm{~cm})$ with a central platform (diameter $35 \mathrm{~cm}$ ). Closed arms had walls of darkened Plexiglass $20 \mathrm{~cm}$ high along their length. Open arms had a 2-cm-high lip along the edges of the arms. Immediately following the elevated plus maze exposure, animals were individually housed and left undisturbed in a quiet holding room for $30 \mathrm{~min}$. Animals were then decapitated, and brains were removed, frozen on dry ice, and stored at $-80^{\circ} \mathrm{C}$.

\section{NEURAL ASSESSMENT OF C-FOS mRNA BY IN SITU HYBRIDIZATION Probes and labeling}

The ribonucleotide probe was prepared using a rat $c$-fos $2116 \mathrm{bp}$ template provided by Dr. Victor Viau (Department Cellular and Physiological Sciences, The University of British Columbia, Canada). Probes were labeled with ${ }^{35}$ S-UTP (Amersham Biosciences, NJ, USA) using Polymerase $\mathrm{T}^{7}$ and Promega Riboprobe System (Promega Corporation, Madison, WI, USA). All probes were purified using Micro Bio-Spin 30 Columns (Bio-Rad, CA, USA). One molar of DTT was added to prevent oxidation.

\section{In situ hybridization}

Brains were sectioned coronally $(20 \mu \mathrm{m})$ using a cryostat $\left(-16^{\circ} \mathrm{C}\right)$ and stored at $-80^{\circ} \mathrm{C}$. Thawed sections were fixed in formalin for $30 \mathrm{~min}$ and then pre-hybridized as follows: $1 \times$ PBS twice for $10 \mathrm{~min}$ each, proteinase $\mathrm{K}\left(100 \mu \mathrm{g} / \mathrm{L}\right.$; at $\left.37^{\circ} \mathrm{C}\right)$ for $9 \mathrm{~min}$, $0.1 \mathrm{M}$ triethanolamine-hydrochloride (TEA) for $10 \mathrm{~min}, 0.1 \mathrm{M}$ TEA with $0.25 \%$ acetic anhydride for $10 \mathrm{~min}, 2 \times$ SSC twice for $10 \mathrm{~min}$ each, dehydration by a graded series of ethanol, chloroform for $5 \mathrm{~min}$, and finally 100\% ethanol before being air dried. Hybridization buffer (75\% formamide, $3 \times$ SSC, $1 \times$ Denhardt's solution, $200 \mu \mathrm{g} / \mathrm{mL}$ yeast tRNA, $50 \mathrm{mM}$ sodium phosphate buffer ( $\mathrm{pH} 7.4), 10 \%$ dextran sulfate, and $10 \mathrm{mM}$ DTT) was applied $\left(1 \times 10^{6} \mathrm{cpm} / \mathrm{slide}\right)$ and covered with HybriSlips (Sigma-Aldrich, $\mathrm{ON}$, Canada). Sections were incubated overnight at $55^{\circ} \mathrm{C}$ in chambers humidified with $75 \%$ formamide. HybriSlips were removed and the slides were rinsed as follows: $2 \times$ SSC twice for $20 \mathrm{~min}, 2 \times$ SSC for $30 \mathrm{~min}, 50 \mu \mathrm{g} / \mathrm{L}$ RNAse A solution (at $37^{\circ} \mathrm{C}$ ) for $60 \mathrm{~min}$, $2 \times$ SSC with $0.01 \mathrm{M} \mathrm{DTT}$ for $10 \mathrm{~min}, 1 \times$ SSC for $10 \mathrm{~min}, 0.5 \times$ SSC with $0.01 \mathrm{M} \mathrm{DTT}$ for $10 \mathrm{~min}, 0.1 \times \mathrm{SSC}$ with $0.01 \mathrm{MDTT}\left(\right.$ at $60^{\circ} \mathrm{C}$ ) for $60 \mathrm{~min}$, and $0.1 \times$ SSC for $5 \mathrm{~min}$. Sections were dehydrated by a graded series of ethanol and air dried overnight.

Kodak BioMax autoradiography film was exposed to hybridized slides of the ventral hippocampus for 28 days, and then developed using Kodak GBX developer and fixer. For all other areas, the hybridized slides were dipped in Kodak NTB2 autoradiography emulsion (diluted 50:50 with distilled water) and exposed in desiccated sealed, light tight boxes $\left(4^{\circ} \mathrm{C}\right)$ for 49 days for $\mathrm{mPFC}$, 70 days for mpdPVN, and 92 days for the amygdala. Slides were developed using Kodak D19 developer and standard Kodak fixer, counterstained with Toluidine Blue, and coverslipped with Permount (Fisher Scientific Ltd.). For all brain areas analyzed, $n=4-5$ with the exception of the ventral hippocampal formation (CA1, CA3, DG, and ventral subiculum) where $n=3$ for PF non-CMS males.

\section{Densitometric analysis}

The autoradiographic films for the ventral hippocampal formation were scanned and analyzed with Scion Image 4.0.3.2 (National Institutes of Health, USA). The left and right DG, CA1, and CA3, and ventral subiculum were traced freehand according to a stereotaxic rat brain atlas (46) in two sections per animal to determine mean gray density levels. Background was measured from the corpus callosum, and corrected gray levels were obtained by subtracting the background level from each of the four measurements. Left and right levels in each measured area were averaged together for analysis. For the emulsion dipped slides (amygdala, mPFC, and 
mpdPVN), in situ signals were visualized with a Q-imaging monochrome 12-bit camera attached to a Zeiss Axioskop 2 motorized plus microscope. Images were captured under dark field illumination using Northern Elite 6.0v (Empix Imaging, Inc., Mississauga, ON, Canada) and analyzed with ImageJ 10.3 software (National Institutes of Health, USA).

\section{STATISTICAL ANALYSIS}

Data were first analyzed using two-way analysis of variance (ANOVA for the factors of prenatal treatment and CMS exposure) followed by Newman-Keuls post hoc tests for each brain region (presented in Figures 1-4). Differences were considered significant at $p \leq 0.05$. Further analyses utilized planned comparisons to test the a priori hypotheses that: (1) PAE will alter the response to acute stress, i.e., non-CMS PAE animals will show differential neural activity in response to acute stress (exposure to the elevated plus maze) compared to non-CMS control animals; and (2) CMS will differentially alter neural activity in response to acute stress in PAE compared to control animals.

In order to identify networks of brain regions altered by our experimental conditions, we employed CPCA, which is performed in two steps, referred to as the external analysis and the internal analysis. The external analysis consists of multivariate leastsquares multiple regression, which serves to separate the overall variance (i.e., $c$-fos mRNA activity in the 13 brain regions of interest) into the variance that can (and cannot) be predicted by the experimental conditions (i.e., prenatal treatment and CMS exposure). This first step of CPCA simply separates the predicted and residual scores through multivariate multiple regression, using dummy-coded groupings as the independent variables [ 1 for group membership, 0 for not; (36)]. This first step produces a matrix of predicted scores that reflects the portion of variance in brain neural activity that is attributable to the experimental conditions (i.e., the predictable variance). A matrix of residual scores is also produced, which reflects the portion of variance in brain neural activity that is not attributable to experimental conditions (i.e., the residual variance). This residual variance was not further analyzed for the present study, as we were interested in brain networks associated with our experimental conditions. The second step in CPCA, the internal analysis, simply consists of a PCA on the predictable variance (i.e., the predicted scores from the multivariate regression in the first step). PCA is a data reduction technique that serves to combine large sets of related variables by reducing them to a smaller number of components (referred to as networks when the dependent variables consist of brain regions) that best explain the variance, while losing as little information as possible. The components that emerge from the PCA on the predicted scores reflect the brain networks that characterize the differences in brain activity between the experimental conditions (i.e., the brain networks that show differing degrees of activity across the different prenatal treatment and CMS groups). All PCA solutions were separately rotated using Varimax with Kaiser normalization, and the number of the components extracted was determined by inspection of scree plots $(47,48)$. In order to examine specifically how the groups differ in terms of brain activity in each of the resulting networks, correlations were computed between the experimental groups and the component scores from each of the extracted components. A more detailed description of the theory
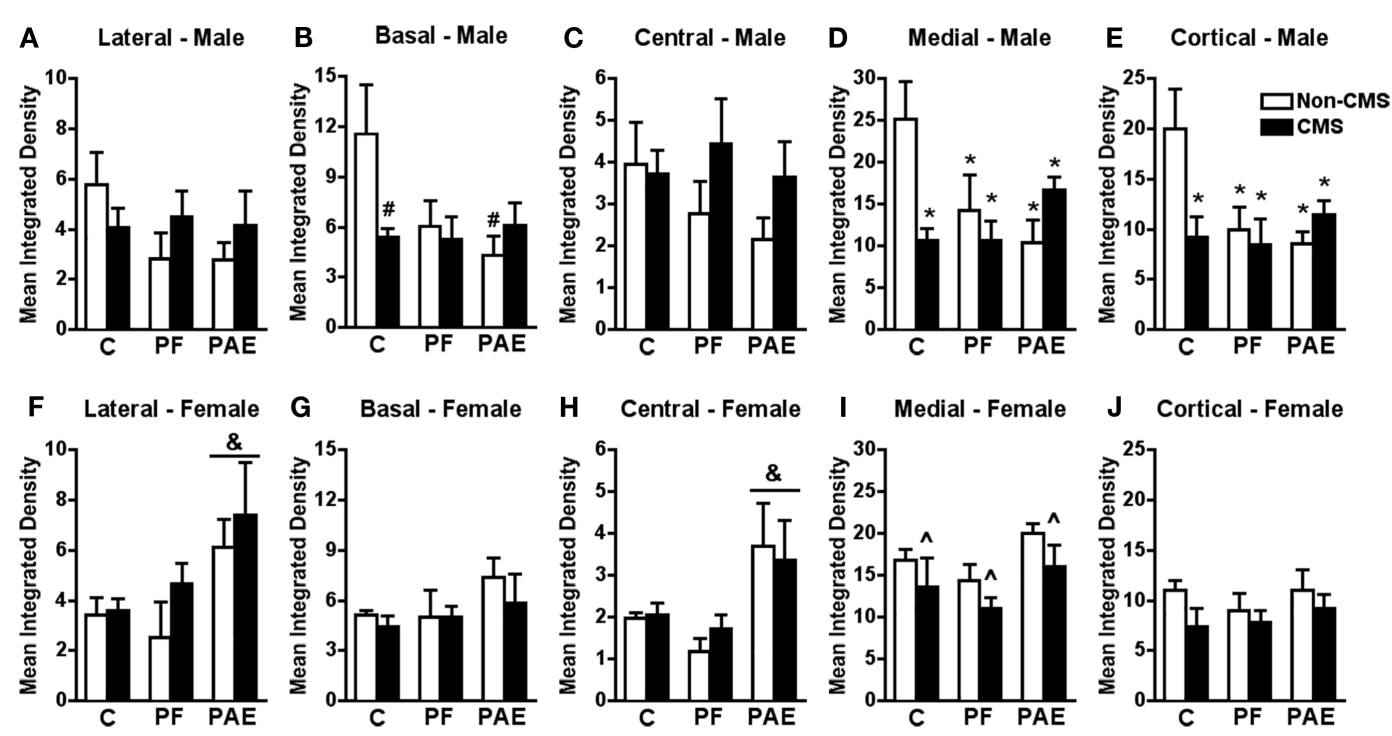

FIGURE 1 | Amygdala $c$-fos mRNA expression in response to the elevated plus maze test in male and female control (C), pair-fed (PF), and prenatal alcohol exposed (PAE) rats, with or without exposure to chronic mild stress (CMS) in adulthood. Bars represent the integrated density (mean \pm SEM) of $c$-fos mRNA expression in the lateral $(\mathbf{A}, \mathbf{F})$, basal (B,G), central (C,H), medial (D,I), and cortical (E,J) amygdala nuclei.

* Indicates a significant interaction between prenatal treatment and CMS exposure where all groups are different from $\mathrm{C}$ non-CMS; \&indicates a significant main effect of prenatal treatment where the post hoc test shows that PAE is different from $\mathrm{C}$ and PF, independent of $\mathrm{CMS}$; ^ indicates a significant main effect of CMS exposure where all animals exposed to CMS are different from animals not exposed to CMS; \#indicates that control CMS and PAE non-CMS are different from control non-CMS based on a priori comparisons ( $n=4-5$ for all groups). 
A

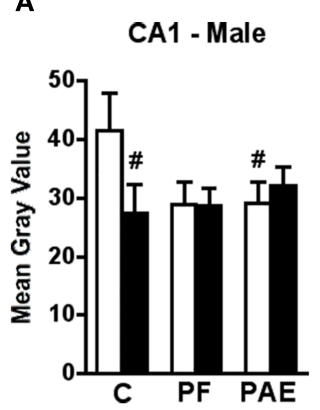

E

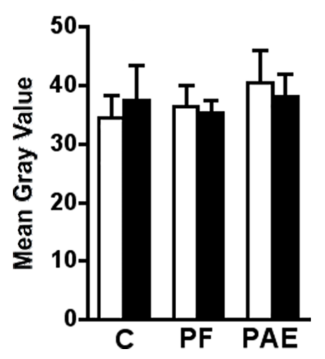

B

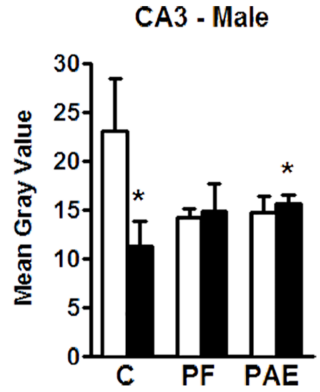

$\mathbf{F}$

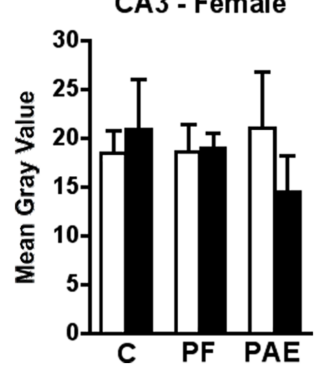

C

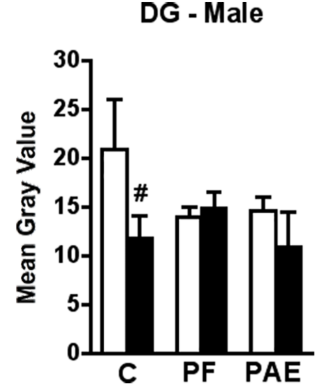

G

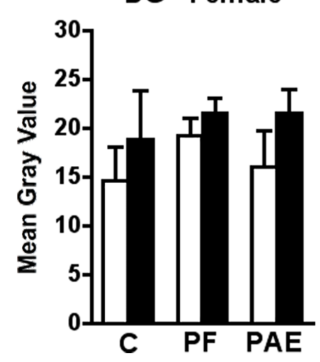

D

\section{Ventral Subiculum - Male}

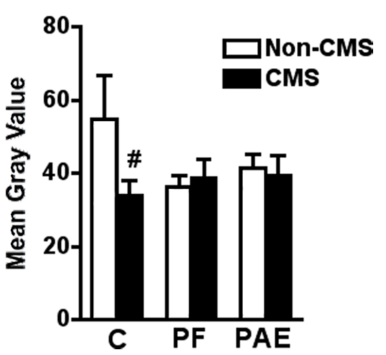

H

Ventral Subiculum - Female

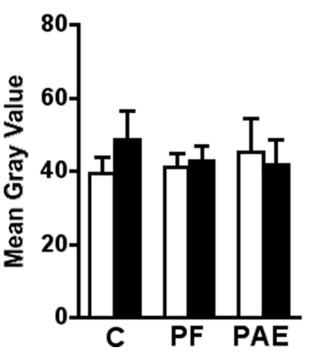

FIGURE 2 | Hippocampal formation $c$-fos mRNA expression in response to the elevated plus maze test in male and female control (C), pair-fed (PF), and prenatal alcohol exposed (PAE) rats, with or without exposure to chronic mild stress (CMS) in adulthood. Bars represent the gray value (mean \pm SEM) of $c$-fos mRNA expression in the CA1 (A,E), CA3 (B,F), DG
(C,G), and ventral subiculum (D,H). * Indicates a significant interaction between prenatal treatment and CMS exposure, where the control non-CMS group is different from control CMS and PAE CMS groups; "indicates that control CMS and PAE non-CMS are different from control non-CMS based on a priori comparisons ( $n=3-5$ for all groups). and methodology of CPCA can be found in previously published manuscripts (38-41, 49, 50). CPCA was carried out using SPSS.

\section{RESULTS \\ AMYGDALA \\ Males}

$c$-fos mRNA expression within the medial and cortical amygdala nuclei in response to acute stress was significantly reduced in non-CMS PAE and PF compared to non-CMS control males (Figures 1D,E). Moreover, while CMS reduced $c$-fos mRNA expression within the medial and cortical amygdala nuclei in control males compared to that in their non-CMS counterparts, $c$-fos mRNA expression of PAE and PF rats was similar under CMS and non-CMS conditions following acute stress \{significant interaction between prenatal treatment and CMS exposure for medial $\left[F_{(1,23)}=6.578 ; p<0.007\right]$ and cortical $\left[F_{(1,23)}=4.196 ; p<0.03\right]$ amygdala nuclei\}.

Similarly, $c$-fos mRNA expression within the basal amygdala in response to acute stress was significantly reduced in non-CMS PAE compared to non-CMS control males (Figure 1B). Moreover, CMS control males showed reduced $c$-fos mRNA expression within the basal nucleus compared to that in their non-CMS counterparts, whereas PAE rats were similar in $c$-fos mRNA expression following acute stress under both CMS and non-CMS conditions no significant interaction between prenatal treatment and CMS interaction $\left[F_{(1,23)}=3.129 ; p=0.06\right]$; a priori analysis indicated that PAE non-CMS $(p<0.005)$ and control CMS $(p<0.02)$ showed lower $c$-fos mRNA expression than the control non-CMS $\}$.
Neither prenatal treatment nor CMS significantly altered c-fos mRNA expression within the central or lateral amygdala nuclei following acute stress $\{$ Figures 1A,C; no significant interaction between prenatal treatment and CMS exposure for central $\left[F_{(1,22)}=0.803\right]$ and lateral $\left[F_{(1,23)}=1.499\right]$ amygdala nuclei\}.

\section{Females}

Among females, a different pattern of neural activity within the amygdala was observed. PAE, independent of CMS exposure, increased $c$-fos mRNA expression within the central and lateral amygdala nuclei in response to acute stress when compared to that in control and PF animals \{Figures 1F,H; no significant interaction between prenatal treatment and CMS exposure for central $\left[F_{(1,23)}=0.448\right]$ or lateral $\left[F_{(1,23)}=0.387\right]$ amygdala nuclei, but significant main effect of prenatal treatment for central $\left[F_{(2,23)}=7.198 ; p<0.005\right]$ and lateral $\left[F_{(2,23)}=4.910 ; p<0.02\right]$ nuclei in response to acute stress\}.

In the medial amygdala, CMS reduced $c$-fos mRNA expression in response to acute stress independent of prenatal treatment group \{Figure 1I; no significant interaction between prenatal treatment and CMS exposure $\left[F_{(1,23)}=0.015\right]$, but significant main effect for CMS $\left.\left[F_{(1,23)}=4.176 ; p=0.05\right]\right\}$.

Neither prenatal treatment nor CMS significantly altered $c$-fos mRNA expression in response to acute stress within the cortical and basal amygdala nuclei \{Figures 1G,J; no significant interaction between prenatal treatment and CMS exposure for cortical $\left[F_{(1,23)}=0.312\right]$ or basal $\left[F_{(1,23)}=0.257\right]$ amygdala nuclei $\}$. 

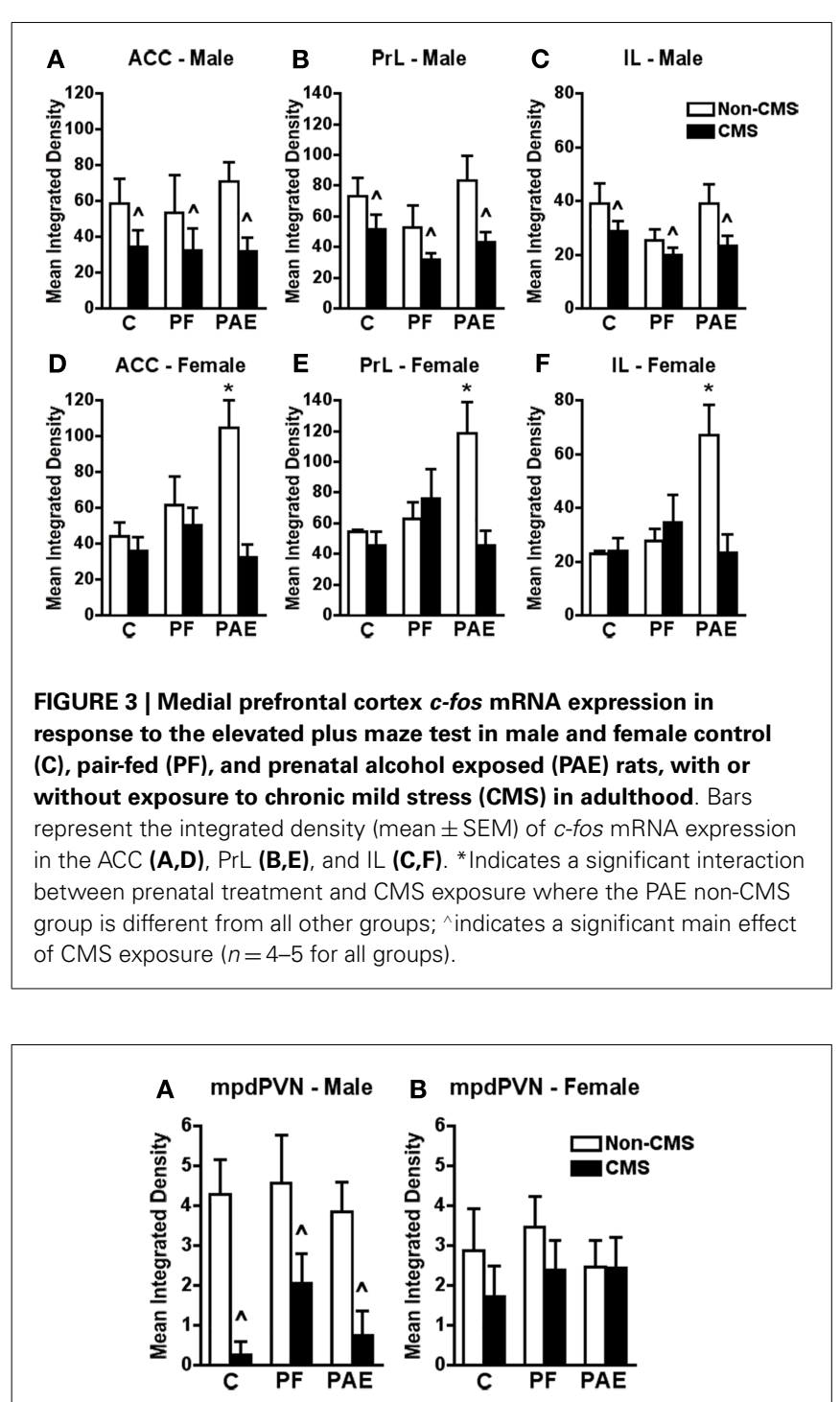

FIGURE 4 | Medial parvocellular dorsal division of the paraventricular nucleus of hypothalamus $c$-fos mRNA expression in response to the elevated plus maze test in male (A) and female (B) control (C), pair-fed (PF), or prenatal alcohol exposed (PAE) rats, with or without exposure to chronic mild stress (CMS) in adulthood. Bars represent the integrated density (mean \pm SEM) of $c$-fos mRNA expression in the mpdPVN.

$\wedge$ Indicates a significant main effect of CMS exposure ( $n=4-5$ for all groups).

\section{HIPPOCAMPAL FORMATION \\ Males}

$c$-fos mRNA expression within the CA3 subregion of the ventral hippocampal formation in response to acute stress was significantly reduced in CMS PAE compared to non-CMS control males (Figure 2B). Moreover, similar to the medial and cortical amygdala, while CMS reduced $c$-fos mRNA expression within the $\mathrm{CA} 3$ region in control males compared to their non-CMS counterparts, $c$-fos mRNA expression of PAE and PF rats was similar under CMS and non-CMS conditions in response to acute stress \{significant interaction between prenatal treatment and CMS exposure $\left.\left[F_{(1,19)}=3.714 ; p<0.05\right]\right\}$.
Consistent with these findings, $c$-fos mRNA expression in response to acute stress within the CA1 subregion of the ventral hippocampal formation was significantly reduced in non-CMS PAE compared to non-CMS control males (Figure 2A). Moreover, while $c$-fos mRNA expression was reduced within the CA1, DG, and ventral subiculum subregions in CMS control compared to non-CMS control males, $c$-fos mRNA expression of PAE rats was similar under CMS and non-CMS conditions in response to acute stress \{Figures 2A,C,D; no significant interaction between prenatal treatment and CMS interaction for CA1 $\left[F_{(1,20)}=2.257\right]$, DG $\left[F_{(1,20)}=1.472\right]$, or ventral subiculum $\left[F_{(1,19)}=1.839\right]$; a priori analysis indicates that non-CMS PAE showed a significantly lower $c$-fos mRNA expression within the CA1 $(p=0.05)$ than non-CMS controls, and that CMS controls $(p<0.03)$ showed lower $c$-fos mRNA expression than non-CMS control within CA1, DG, and ventral subiculum\}.

\section{Females}

Among females, neither prenatal treatment nor CMS significantly altered $c$-fos mRNA expression in response to acute stress within any subregion of the hippocampal formation \{Figures $2 \mathbf{E}-\mathbf{H}$; no significant interaction between prenatal treatment and CMS exposure for CA1 $\left[F_{(1,21)}=0.193\right]$, CA3 $\left[F_{(1,21)}=0.399\right]$, DG $\left[F_{(1,21)}=0.130\right]$, or ventral subiculum $\left.\left[F_{(1,21)}=0.490\right]\right\}$.

\section{MEDIAL PREFRONTAL CORTEX Males}

In all mPFC subregions (ACC, PrL, and IL), CMS reduced $c$ fos mRNA expression in response to acute stress independent of prenatal treatment \{Figures $3 \mathrm{~A}-\mathrm{C}$; no significant interaction between prenatal treatment and CMS exposure for ACC $\left[F_{(1,22)}=0.299\right]$, PrL $\left[F_{(1,22)}=0.474\right]$, or IL $\left[F_{(1,22)}=0.421\right]$, but significant main effect for CMS for ACC $\left[F_{(1,22)}=7.426\right.$; $p<0.02]$, PrL $\left[F_{(1,22)}=8.936 ; p<0.008\right]$, and IL $\left[F_{(1,22)}=5.365\right.$; $p<0.03]\}$.

\section{Females}

Similar to findings for the amygdala, $c$-fos mRNA expression in response to acute stress within all areas of the $\mathrm{mPFC}$ was significantly increased in non-CMS PAE females compared to non-CMS control and PF females (Figures 3D-F). Moreover, while CMS PAE females showed reduced $c$-fos mRNA expression within the ACC, PrL, and IL compared to non-CMS PAE females, $c$-fos mRNA expression of control and PF rats was similar under CMS and non-CMS conditions in response to acute stress \{significant interaction between prenatal treatment and CMS exposure for ACC $\left[F_{(1,23)}=4.614 ; p<0.03\right], \operatorname{PrL}\left[F_{(1,23)}=5.139 ; p<0.02\right]$, and IL $\left.\left[F_{(1,23)}=6.525 ; p<0.007\right]\right\}$.

\section{MEDIAL PARVOCELLULAR DORSAL DIVISION OF THE PARAVENTRICULAR NUCLEUS OF HYPOTHALAMUS Males}

In the mpdPVN, CMS reduced $c$-fos mRNA expression in response to acute stress independent of prenatal treatment \{Figure 4A; no significant interaction between prenatal treatment and CMS exposure $\left[F_{(1,23)}=0.257\right]$, but significant main effect for CMS $\left.\left[F_{(1,23)}=15.541 ; p<0.001\right]\right\}$. 


\section{Females}

By contrast to males, neither prenatal treatment nor CMS significantly altered $c$-fos mRNA expression among females within mpdPVN in response to acute stress \{Figure $4 \mathrm{~B}$; no significant interaction between prenatal treatment and CMS exposure $\left.\left[F_{(1,23)}=0.783\right]\right\}$.

\section{CONSTRAINED PRINCIPAL COMPONENT ANALYSIS Males}

The experimental conditions (i.e., prenatal treatment and CMS exposure) accounted for $34.17 \%$ of the total variance in $c$-fos mRNA expression in males. PCA on this constrained variance (i.e., the predictable variance) revealed a two-component solution; Table 1 displays the component loadings for each of the 13 brain regions on each component. The first component explained $19.40 \%$ of the total variance and $56.78 \%$ of the predictable variance and was defined as Amygdala + Hippocampal Formation because the medial, cortical, and basal amygdala nuclei, and CA3 and CA1 hippocampal regions showed the highest loadings, with lesser contributions from the ventral subiculum, DG, and lateral amygdala (Figure 5B). The second component explained $11.26 \%$ of the total variance and $32.97 \%$ of the predictable variance and was defined as Prefrontal Cortex + Paraventricular Nucleus because the PrL, mpdPVN, and ACC showed the highest loadings, with lesser contributions from the IL and central amygdala (Figure 5D).

Correlations between the subjects' component scores and their experimental condition membership are displayed in Figure 5A for the Amygdala + Hippocampal Formation network and in Figure 5C for the Prefrontal Cortex + Paraventricular Nucleus network. The Amygdala + Hippocampal Formation network showed a significant positive correlation with the control non-CMS condition $(r=0.96, p<0.0001)$, but a significant negative correlation with the PAE non-CMS condition $(r=-0.39, p<0.05)$. Furthermore, although not reaching statistical significance, the Amygdala + Hippocampal Formation network was also negatively correlated with the control CMS condition. As seen in Figure 5C, the Prefrontal Cortex + Paraventricular Nucleus network showed positive correlations with all non-CMS conditions; however only PAE non-CMS $(r=0.79, p<0.0001)$ reached significance. Conversely, the Prefrontal Cortex + Paraventricular Nucleus network showed negative correlations with all CMS groups, with PAE $(r=-0.44$, $p<0.05)$ and PF $(r=-0.45, p<0.05)$ CMS conditions showing significant correlations.

\section{Females}

The experimental conditions accounted for $25.72 \%$ of the total variance in $c$-fos expression in females. PCA on this constrained (i.e., predictable) variance revealed a two-component solution (see Table 2 for component loadings). The first component explained $12.04 \%$ of the total variance and $46.81 \%$ of the predictable variance and was defined as Prefrontal Cortex because all mPFC subregions (ACC, IL, and PrL) contributed with the highest loadings to this component (Table 2; Figure 6B). The second component explained $9.14 \%$ of the total variance and $35.56 \%$ of the predictable variance and was defined as Amygdala because the majority of amygdala nuclei (central, lateral, medial, and basal)
Table 1 | Component loadings for the predicted solution in males.

\begin{tabular}{lcc}
\hline Variables & $\begin{array}{l}\text { Amygdala }+ \\
\text { Hippocampal } \\
\text { Formation }\end{array}$ & $\begin{array}{l}\text { Prefrontal Cortex }+ \\
\text { Paraventricular } \\
\text { Nucleus }\end{array}$ \\
\hline Medial amygdala & $\mathbf{0 . 6 5}$ & 0.06 \\
Cortical amygdala & $\mathbf{0 . 6 3}$ & 0.09 \\
CA3 & $\mathbf{0 . 6 1}$ & 0.17 \\
Basal amygdala & $\mathbf{0 . 5 8}$ & 0.04 \\
CA1 & $\mathbf{0 . 5 3}$ & 0.09 \\
Ventral subiculum & $\mathbf{0 . 4 8}$ & 0.20 \\
DG & $\mathbf{0 . 4 4}$ & 0.24 \\
Lateral amygdala & $\mathbf{0 . 3 7}$ & -0.17 \\
Prelimbic & 0.14 & $\mathbf{0 . 5 9}$ \\
Paraventricular nucleus & 0.12 & $\mathbf{0 . 5 4}$ \\
Anterior cingulate & 0.07 & $\mathbf{0 . 5 4}$ \\
Infralimbic & 0.22 & $\mathbf{0 . 4 8}$ \\
Central amygdala & 0.21 & $-\mathbf{0 . 3 6}$ \\
\hline
\end{tabular}

Values $\geq 0.30$ are set in bold.

contributed with the highest loadings to this component; however, the IL also contributed to this component (Table 2; Figure 6D).

Correlations between the subjects' component scores and their experimental condition membership are displayed graphically in Figure 6A for the Prefrontal Cortex network and in Figure 6C for the Amygdala network. The Prefrontal Cortex network showed a significant positive correlation with the PAE non-CMS condition $(r=0.82, p<0.0001)$. In contrast, the Prefrontal Cortex network showed a significant negative correlation with the PAE CMS condition $(r=-0.64, p<0.0001)$. Additionally, as seen in Figure 6C, the Amygdala network showed a significant positive correlation with the PAE group, independent of CMS condition $(r=0.56, p<0.01$ for PAE non-CMS and $r=0.67, p<0.0001$ for PAE CMS); however, this network was negatively correlated with the control group, again, independent of CMS condition. Furthermore, the Amygdala network showed a significant negative correlation with the PF non-CMS condition $(r=-0.55, p<0.01)$.

\section{DISCUSSION}

Stress and emotional regulation are dynamic and complex processes achieved by the fine coordination of interconnected brain regions, including, but not limited to, the amygdala, mPFC, hippocampus, and paraventricular nucleus of hypothalamus (PVN) (14-17, 51). The majority of these brain areas have dual functions, playing major roles in both the stress response and emotional regulation. Because of these dual roles and the intrinsic interconnectivity among these brain regions, dysfunction within or among structures in this neurocircuitry could result in dysregulation of the stress response and/or lead to mood and anxiety disorders. The present results indicate that, regardless of chronic stress later in life, PAE produces widespread alterations in neural activity within and between several brain areas of the stress/emotional neurocircuitry analyzed. Notably, male and female rats in the current study were differentially affected by PAE. Overall, PAE decreased neural activity within the amygdala and hippocampal formation in males and increased neural activity 


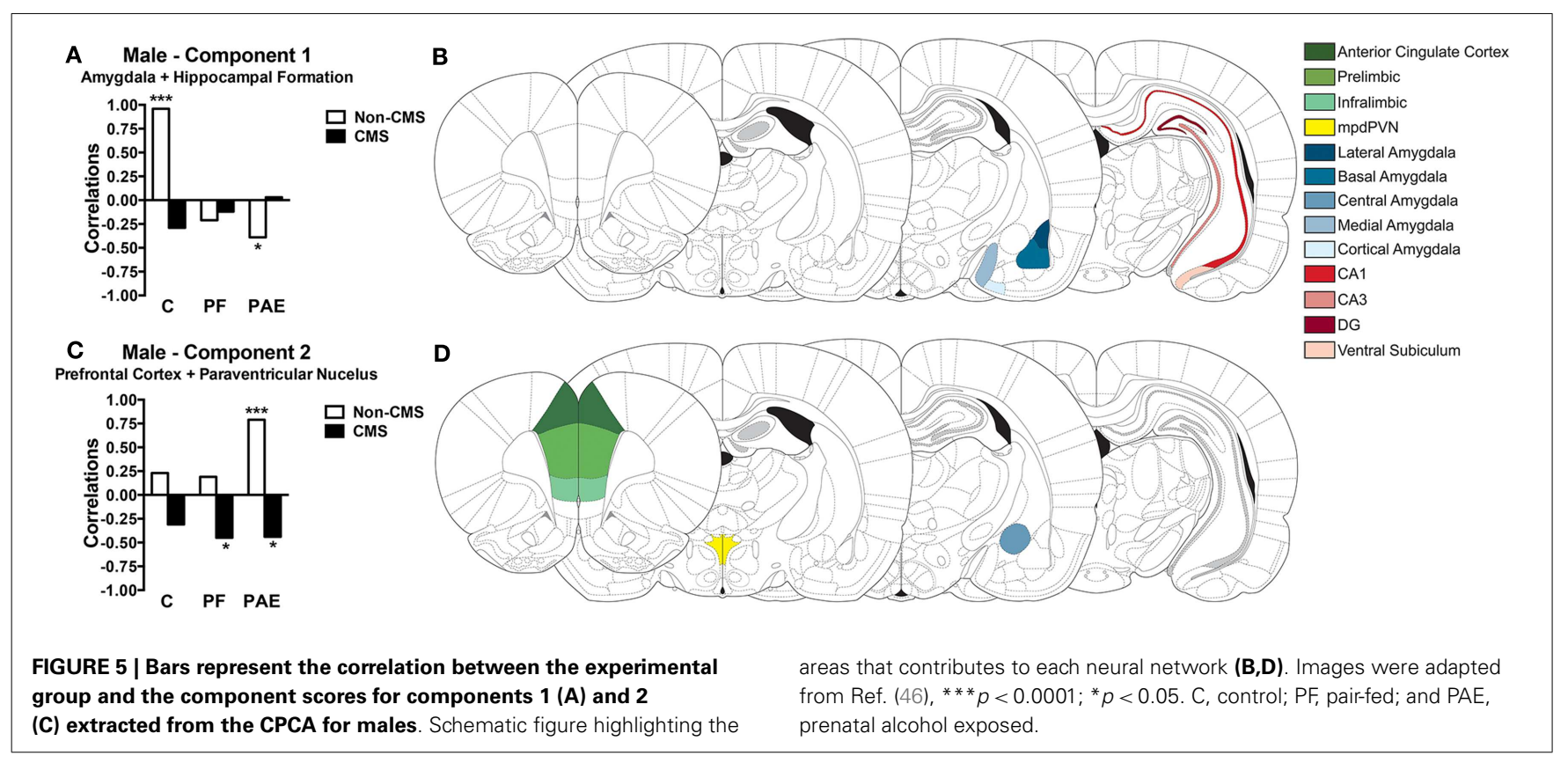

Table 2 | Component loadings for the predicted solution in females.

\begin{tabular}{lcc}
\hline Variables & Prefrontal Cortex & Amygdala \\
\hline Anterior cingulate & $\mathbf{0 . 6 9}$ & 0.20 \\
Infralimbic & $\mathbf{0 . 6 2}$ & $\mathbf{0 . 3 5}$ \\
Prelimbic & $\mathbf{0 . 6 1}$ & 0.24 \\
Cortical amygdala & 0.21 & 0.15 \\
CA3 & 0.20 & -0.09 \\
DG & -0.23 & 0.01 \\
Central amygdala & 0.12 & $\mathbf{0 . 6 0}$ \\
Lateral amygdala & -0.06 & $\mathbf{0 . 5 6}$ \\
Medial amygdala & 0.29 & $\mathbf{0 . 3 4}$ \\
Basal amygdala & 0.26 & $\mathbf{0 . 3 1}$ \\
CA1 & 0.10 & 0.17 \\
Paraventricular nucleus & 0.00 & -0.15 \\
Ventral subiculum & 0.03 & 0.03 \\
\hline
\end{tabular}

Values $\geq 0.30$ are set in bold.

within the amygdala and mPFC in females. Exposure to CMS also resulted in a sexually dimorphic effect, as it reduced neural activity within the mPFC and PVN in PAE males, but reduced activity in all areas analyzed in controls. Conversely, CMS reduced neural activity only in the mPFC of PAE females and had no effects in control females. Importantly, these patterns of neural activity were reflected in the differential networks of brain activation for PAE and control male and female rats. Indeed, the CPCA revealed that, contrary to non-CMS PAE males, non-CMS control males showed a strongly active Amygdala + Hippocampal Formation network. Conversely, non-CMS PAE males showed a strongly active Prefrontal Cortex + Paraventricular Nucleus network. Among females, non-CMS PAE animals showed strongly active Prefrontal Cortex and Amygdala networks, which contrasts with the network activity observed in non-CMS controls. Of note, CMS also altered the networks of brain activation in PAE animals, as the pattern of activity within the Prefrontal Cortex + Paraventricular Nucleus network in PAE males and the Prefrontal Cortex network in PAE females switched from being active in the non-CMS animals to showing decreased activation in CMS animals. Overall, the changes in the network of brain areas following PAE could be implicated in the hyperresponsivity to stress and increased vulnerability to the development of psychopathologies observed among individuals prenatally exposed to alcohol $(5,9,11-13,22,23)$.

\section{AMYGDALA}

Clinical studies have consistently demonstrated that amygdala dysfunction is associated with mood and anxiety disorders $(16,17,52$, 53). These disorders are among the most prevalent mental health problems in children and adults exposed to alcohol during gestation (11-13). Animal models have supported the relationship between amygdala dysfunction and anxiety- and depressive-like behaviors (52-55). Specifically, the basal and lateral amygdala nuclei have been defined as part of the neural circuitry activated in animals exhibiting depressive-like behaviors $(56,57)$. Additionally, the central, lateral, and basal amygdala nuclei have a large population of neurons that express corticotropin-releasing hormone $(\mathrm{CRH})$ and its receptors $(58,59)$; hyperfunction of these neurons can result in increased levels of anxiety-like behaviors $(60,61)$.

The present results indicate sexually dimorphic effects of PAE and/or CMS on neural activity of the amygdala nuclei. PAE females showed an increase in neural activity within the central and lateral amygdala nuclei in response to acute stress, regardless of whether they were exposed to CMS. Increased neural activity in the amygdala is consistent with the increased stress responsiveness as well as anxiety-/depressive-like behavior often seen in PAE females (5, 31,32 ). However, vulnerability to anxiety-/depressive-like behavior is often greater in PAE animals following CMS than in their non-CMS counterparts, and under some conditions, non-CMS 


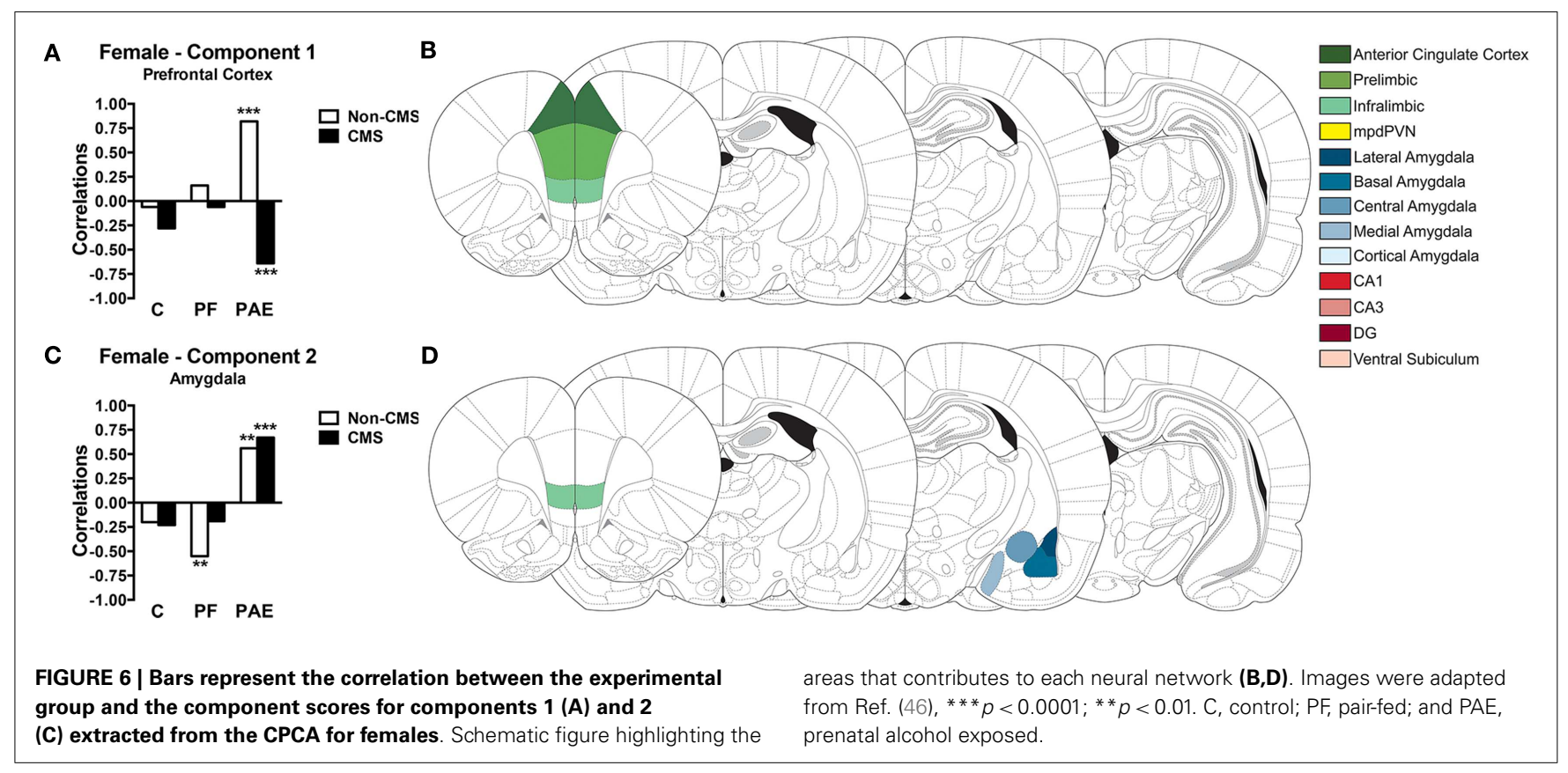

PAE animals may not differ from non-CMS controls. We found, for example, that while non-CMS PAE females were similar to non-CMS controls in time spent on the open arms of an elevated plus maze, exposure to CMS significantly reduced frequency of total open arm entries for PAE but not control females (31). These discrepancies in neural activity and behavior suggest that other brain areas besides the amygdala may be modulating anxiety-like behavior among PAE females. In males, PAE and pair-feeding prevented the increase in neural activity within the medial, cortical, and basal amygdala nuclei shown by control non-CMS animals. However, compared to controls, neither PAE nor CMS changed the neural activity of central and lateral amygdala nuclei in response to acute stress. These data are interesting in light of the finding that PAE males exposed to CMS show more robust increases in anxiety-like behavior in the elevated plus maze than PAE females (31), suggesting that the neural mechanisms involved in mediating the anxiety response among males may be dissociable from those in females. This is supported by our finding of networks of brain regions that were differentially associated with PAE and/or CMS in males and females in the current study, as discussed further below.

In addition to its well-characterized role in emotional processing, the amygdala also regulates different aspects of the neuroendocrine stress response $(15,62)$. In general, the central nucleus of the amygdala regulates the HPA response to systemic stressors, and integrates autonomic responses to psychogenic stressors; the basal, lateral, and the medial amygdala nuclei regulate the HPA response to psychological stressors (62). The increased neural activity within the lateral amygdala of PAE females reported here may, at least partially, underlie the hyperresponsivity to stress observed in these animals $(5,9,22,23)$.

\section{HIPPOCAMPAL FORMATION}

The hippocampal formation is well known for its role in learning and memory, stress, and emotional regulation (63). However, the dorsal and ventral components of the hippocampal formation have different functions: the dorsal hippocampus is primarily involved in cognitive functions while the ventral hippocampus is more essential for stress and emotional regulation (63). For example, lesions to the ventral hippocampus result in decreased anxietylike behaviors $(63,64)$, whereas increased activity in the ventral hippocampus is positively correlated with anxiety-like behavior $(65,66)$. Additionally, the hippocampal formation is consistently associated with an overall inhibition of the stress response $(14,15)$. Indeed, hippocampal lesions result in increased stress responses and, in some cases, increased basal levels of corticosterone (67). Stimulation of the CA3, DG, and ventral subiculum subfields, on the other hand, results in reduced glucocorticoid secretion (68). Interestingly, PAE is known to induce morphological changes that can lead to functional deficits in the hippocampus: studies in the clinical literature reveal reduced hippocampal volume in individuals exposed to alcohol during gestation $(20,21)$. In rats, PAE results in hypertrophy of mossy fibers (69), reduced numbers of dendritic spines (70), as well as impaired long-term potentiation $(71,72)$ and adult neurogenesis - primarily in males $(73,74)$. In agreement with these findings, our results indicate that PAE males show reduced neural activity in the hippocampal formation in response to acute stress when compared to controls. This deficit in neural activity suggests that the inhibitory action of the hippocampal formation may be reduced, which could contribute to the hyperresponsivity to stress, and ultimately to the increase in anxiety-like behaviors observed in PAE animals (31).

Interestingly, PAE results in no apparent changes in basal expression of glucocorticoid and mineralocorticoid receptors in the hippocampal formation of male animals (75-77), whereas in females, some studies indicate a reduction (77), whereas others report no change $(75,76)$ in basal expression of glucocorticoid and mineralocorticoid receptor mRNA. However, removal of the inhibitory feedback effects of corticosterone on the HPA axis by 
adrenalectomy unmasked alterations in basal HPA regulation not apparent in intact animals. Glucocorticoid receptor expression was differentially increased in the hippocampus of PAE males (75) whereas mineralocorticoid receptor expression was differentially increased in the hippocampus of PAE females compared to their control counterparts (75). Moreover, corticosterone replacement was shown to be ineffective at normalizing the adrenalectomyinduced increase in hippocampal mineralocorticoid mRNA levels in PAE males. Together, these results indicate that altered basal regulation of the HPA axis may play a critical role in the hyperresponsivity to stress observed in individuals exposed to alcohol during the prenatal period.

Our results also indicate that CMS reduced the neural activity in the hippocampal formation of control males. This is not surprising given the previous finding that CMS can reduce the number of hippocampal granule cells (78), which could be related to reduced survival of newborn cells (79).

\section{MEDIAL PREFRONTAL CORTEX}

The mPFC also plays a dual role in modulating stress and emotional responses. Abnormal volume and activation of the PFC are commonly observed in individuals with anxiety and mood disorders $(16,52)$. Importantly, functional neuroimaging studies indicate that individuals exposed to alcohol during gestation show greater blood oxygen level-dependent (BOLD) responses in the PFC when performing a behavioral inhibition task (18), suggesting that the PFC could be responding differentially when challenged. In rodents, reduced function of the mPFC, induced by electrolytic lesions or temporary deactivation, results in variable effects: some authors report decreased anxiety-like behaviors (80$82)$ while others report increased anxiety-like behaviors $(83,84)$. These discrepancies on the effects of the mPFC on anxiety-like behavior may be due to the extent of the lesions/deactivation and on the heterogeneous functions of the mPFC subregions. However, overall dysregulation of the $\mathrm{mPFC}$ can lead to an increased predisposition to psychopathologies. Our results show that PAE and control females exhibit different $\mathrm{mPFC}$ responses to the acute stress. Despite the fact that mPFC neural activity is increased in PAE non-CMS females, their behavioral performance was not different from controls in the elevated plus maze (31). Conversely, the neural activity within the mPFC of PAE males was not different from controls; however, PAE increased the expression of anxiety-like behaviors in male rats (31). This dissociation between behavioral and mPFC neural outcomes likely reflects the complex nature of anxiety- and depressive-like disorders, and suggests that other brain areas besides the mPFC may play a role in supporting increased anxiety-like behaviors observed in PAE animals.

The mPFC also plays a major role in modulating the HPA response to stressful events. However, its role is complex due to the fact that the PrL and ACC subregions are involved in inhibition while the IL is involved in excitation of the HPA axis (15, 85-87). Regardless of these opposing roles, our results show that the neural activity within the PrL and ACC in PAE and control animals follows a pattern similar to that within the IL. Specifically, PAE non-CMS females showed a higher response to acute stress in all mPFC areas than their female counterparts, with CMS reducing this neural activity. For the $\operatorname{PrL}$, it is possible that the reduced neural activity in PAE females exposed to CMS may be associated with a reduction in CRH mRNA expression within this area (77), suggesting that the inhibitory action of the PrL may be reduced following CMS, contributing to the hyperresponsivity to stress in PAE females. Similar to the PrL, the ACC is also associated with inhibition of the HPA axis $(15,85-87)$ and the reduced neural activity of the ACC in PAE females exposed to CMS could also contribute to the hyperresponsivity to stress in those animals. Despite an opposite role, the IL - which is involved in excitation of the HPA axis $(15,85-87)$ - also showed reduced neural activity in PAE females exposed to CMS, which suggests a possible alteration in the excitatory/inhibitory balance within the $\mathrm{MPFC}$ of PAE females.

\section{MEDIAL PARVOCELLULAR DORSAL DIVISION OF THE PARAVENTRICULAR NUCLEUS OF HYPOTHALAMUS}

The mpdPVN is composed of a discrete set of neurons that integrate all direct and indirect stress-related inputs from the amygdala, mPFC, and hippocampal formation in order to launch an appropriate response to stress $(15,62)$. These neurons synthesize and secrete $\mathrm{CRH}$, the primary regulator of the pituitary-adrenal axis (88). Our results indicate that, independent of sex, PAE, and control rats showed similar neural activity in the mpdPVN in response to the acute stress of exposure to the elevated plus maze. However, when facing a noxious stressor, such as shock, or a major challenge to HPA regulation, such as adrenalectomy, PAE has been shown to induce enhanced neural activity within the PVN $(75,89)$. Studies have demonstrated that exposure to the elevated plus maze induces small increases in neural activity within the PVN of control animals when compared to the neural activity of animals exposed to shock (34). These results suggest that when PAE animals are tested under basal conditions, or face a mild stressor such as the elevated plus maze, the PVN is capable of responding appropriately; however, when faced with a more severe stressor such as shock, the PVN launches an inappropriately large response.

\section{CONSTRAINED PRINCIPAL COMPONENT ANALYSIS}

The univariate results above indicate that PAE is associated with changes in neural activity across a wide range of brain regions; however, it is important to examine not only individual brain regions, but also how these regions interact to form networks and whether these networks might be differentially altered by PAE and/or CMS. In order to examine this possibility, we employed CPCA, which allowed for the identification of combinations of brain regions that were affected by PAE. CPCA is an ideal statistical technique for examining networks of brain regions that are related to one or more variables of interest (in the current study, prenatal treatment and CMS exposure) because networks are defined only from the portion of the overall variance that is predictable from these independent variables. As such, it was possible to identify networks of brain regions that were associated with PAE (and CMS) in the current study. Comparison between the univariate and multivariate results shows several similarities in terms of the brain regions affected by PAE and CMS; however, the aggregation of these brain regions into networks through CPCA facilitates interpretation. 


\section{Networks regulating stress and emotion in males}

In males, CPCA revealed two functional networks that were collectively responsible for $34.17 \%$ of the total variation. The first network included the majority of the amygdala nuclei (medial, cortical, basal, and lateral) and all of the hippocampal formation areas analyzed (CA1, CA3, DG, and ventral subiculum). This Amygdala + Hippocampal Formation network was strongly active in control animals that were not exposed to CMS, but showed decreased activation in controls subjected to CMS. These findings suggest that the Amygdala-Hippocampal Formation network is critically involved in stress and emotional regulation in control males, and that exposure to chronic unpredictable stress induces functional changes in this network by preventing typical neural activation of the amygdala and hippocampal formation in response to acute stress. Dysregulation in this network may partially explain the increased susceptibility to the development of psychopathologies and abnormal stress responses in animals subjected to CMS (90-93). Importantly, in contrast to controls, the Amygdala + Hippocampal Formation network showed decreased activation for PAE non-CMS animals, indicating that PAE may alter the typical neural activity in these areas such that PAE animals in the non-CMS conditions look similar to controls in the CMS condition.

The second network extracted from the CPCA included all of the mPFC areas analyzed (PrL, ACC, and IL) and the mpdPVN. This Prefrontal Cortex + Paraventricular Nucleus network was activated to some degree in all prenatal groups in the non-CMS condition, although activation of this network was statistically significant only in the PAE non-CMS group. These data suggest that, independent of prenatal treatment, the mPFC and the mpdPVN form a network of structures that work together in the regulation of stress and emotion in males, but that PAE animals engage this network to a greater degree than control animals. This phenomenon could be a compensatory mechanism for the reduced involvement of the Amygdala + Hippocampal Formation network, as indicated by the negative correlation for PAE non-CMS males with this network. Additionally, all prenatal groups subjected to CMS in adulthood showed reduced activity in the Prefrontal Cortex + Paraventricular Nucleus network following acute stress. This switch between positive and negative associations with this network following exposure to CMS highlights the vulnerability of the mPFC and mpdPVN to chronic and unpredictable stress in adulthood. Indeed, the preclinical literature has demonstrated that the PFC is extremely susceptible to the effects of CMS, as chronic stress results in morphological alterations to $\mathrm{mPFC}$ neurons, including retraction of the apical dendritic branches and spine loss in layer II/III neurons (94-96). Additionally, stress-related morphological changes in the mPFC have been associated with increased anxietyand depressive-like behaviors $(90,97)$ as well as with dysfunction in attentional set shifting and working memory $(98,99)$.

\section{Networks regulating stress and emotion in females}

Similar to males, CPCA indicated two functional neural networks in females. However, these networks were more restricted and only included major subdivisions of the same brain regions. The first network (Prefrontal Cortex) included all areas of the mPFC (ACC, IL, and PrL); the second network (Amygdala) included four nuclei of the amygdala (central, lateral, medial, and basal) and the IL. Together, these two functional neural networks in females accounted for $25.72 \%$ of the total variation, in contrast to $34.17 \%$ accounted for by the two functional networks in males.

The Prefrontal Cortex network was strongly activated in PAE non-CMS females, which contrasts with the decreased activity observed among control non-CMS females. Moreover, exposure to CMS resulted in a negative association for PAE females with the Prefrontal Cortex network, whereas there was no significant change in activation of this network in control females. These results indicate that PAE females rely on the Prefrontal Cortex network more than control females for stress and emotional regulation. This abnormal engagement of the Prefrontal Cortex network in response to acute stress is also observed in PAE males and indicates that alcohol exposure during the gestational period results in dysregulation of mPFC function in both sexes. Additionally, our results suggest that the $\mathrm{mPFC}$ of PAE females is more susceptible to the effects of CMS when compared to controls. The switch between positive and negative associations for the Prefrontal Cortex network following exposure to CMS highlights the vulnerability of the $\mathrm{mPFC}$ to chronic and unpredictable stress.

Similarly, the Amygdala network, which also contains the IL region, showed decreased activity in controls, independent of CMS condition, but was significantly positively activated in PAE females independent of CMS. As the amygdala and the IL subregion of the mPFC activate the HPA axis $(14,15)$, this abnormal increased activity of the Amygdala network following PAE may underlie, at least in part, the hyperresponsivity to stress observed in those animals. Additionally, the activity of the Amygdala network in response to acute stress in control and PAE females was not altered by CMS, indicating that, regardless of the level of activity, the amygdala of controls and PAE females is more resistant to chronic and unpredictable stress.

\section{PAIR-FEEDING}

The PF group is used to control the reduced food intake typically observed in alcohol-consuming dams. However, pair-feeding is an imperfect control procedure as it cannot control for the effects of alcohol on absorption and utilization of nutrients. Additionally, despite receiving optimal nutrition during pregnancy (43), the PF dams are underfed compared to the ad libitum-controls, as they receive a reduced ration matched to that of an alcoholconsuming partner. As a result, they are hungry, and typically consume their entire daily food ration within a few hours of presentation, remaining food deprived for the rest of the day. This pair-feeding regimen is thus a mild prenatal stressor, and its effects on offspring behavioral and physiological responsiveness represent, at least partially, an effect of stress above and beyond the nutritional aspect of receiving a reduced food ration. Our results suggest that PF males showed a pattern of neural activity within the amygdala and hippocampal formation similar to PAE males, while the pattern of neural activity within the mPFC and mdpPVN was similar to both PAE and controls. For females, on the other hand, the pattern of neural activity for PF animals was similar to the pattern displayed by the control group. Taken together, these results suggest that the males may be more susceptible to the effects of pair-feeding than females. However, the only effect exclusively observed in PF animals was a significant negative correlation of the Amygdala network with the non-CMS PF female condition, 
which suggests that stress and emotional regulation rely less on the amygdala in PF than PAE or control females.

\section{SUMMARY AND CONCLUSION}

The results presented here build on and expand our knowledge of the differential neural regulation of stress and emotion in individuals exposed to alcohol during gestation. Importantly, despite a relatively low number of animals in one of the experimental groups, the use of CPCA provided a powerful approach for assessing global changes in functional neural networks in PAE compared to control animals, allowing us to go beyond simple assessments of changes in individual brain areas. Indeed, our results highlight how PAE and control males and females recruit different brain networks in the regulation of stress responses and emotion. Specifically, CPCA indicates that while control males rely more on the amygdala and hippocampal formation, PAE males rely more on the $\mathrm{mPFC}$ and mpdPVN when facing a stressful situation. In contrast, the functional neural networks underlying stress and emotional regulation in females are more restricted, as PAE females rely primarily on the mPFC and amygdala. Additionally, our results indicate that CMS differentially affected the neural networks regulating stress and emotion in PAE and control animals. Indeed, exposure to CMS reduced the activity of the Amygdala + Hippocampal Formation network in control males, but reduced the activity of the Prefrontal Cortex + Paraventricular Nucleus network in PAE males. For females, CMS only reduced the activity of the Prefrontal Cortex network in PAE animals. Together, our results suggest that PAE, regardless of its association with CMS, results in a sexually dimorphic dysregulation of the neurocircuitry that underlies stress and emotional regulation, which may be implicated in the stress hyperresponsivity and increased vulnerability to anxiety and depressive disorders observed among individuals exposed to alcohol during gestation (5, 9, 22, 23, 31). Finally, understanding the underlying neural mechanisms of deficits induced by PAE is a crucial step toward the establishment of specific strategies for treating resultant psychopathologies in these individuals.

\section{ACKNOWLEDGMENTS}

This research was funded by NIH/NIAAA grants R37 AA007789 and R01 AA022460, and NeuroDevNet (Canadian Networks of Centers of Excellence) grant 20R64153 to Joanne Weinberg, and Canadian Foundation of Fetal Alcohol Research (CFFAR) grant to Charlis Raineki and Joanne Weinberg. Kim G. C. Hellemans was funded by awards from the Michael Smith Foundation for Health Research and IMPART (Canadian Institute if Health Research STIHR). Tamara Bodnar was funded by Natural Sciences and Engineering Research Council of Canada (NSERC) graduate scholarship. Todd S. Woodward was funded by career investigator awards from CIHR and the Michael Smith Foundation. Katie M. Lavigne was funded by a CIHR award in partnership with the British Columbia Schizophrenia Society.

\section{REFERENCES}

1. Davidson RJ, McEwen BS. Social influences on neuroplasticity: stress and intervention to promote well-being. Nat Neurosci (2012) 15:689-95. doi:10.1038/ nn.3093
2. Levine S. Primary social relationships influence the development of the hypothalamic-pituitary-adrenal axis in the rat. Physiol Behav (2001) 73:255-60. doi:10.1016/S0031-9384(01)00496-6

3. Cirulli F, Francia N, Berry A, Aloe L, Alleva E, Suomi AJ. Early life stress as a risk for mental health: role of neurotrophins from rodents to non-human primates. Neurosci Biobehav Rev (2009) 33:573-85. doi:10.1016/j.neubiorev.2008. 09.001

4. Murphy VE, Smith R, Giles WB, Clifton VL. Endocrine regulation of human fetal growth: the role of the mother, placenta, and fetus. Endocr Rev (2006) 27:141-69. doi:10.1210/er.2005-0011

5. Hellemans KG, Sliwowska JH, Verma P, Weinberg J. Prenatal alcohol exposure: fetal programming and later life vulnerability to stress, depression and anxiety disorders. Neurosci Biobehav Rev (2010) 34:791-807. doi:10.1016/j.neubiorev. 2009.06.004

6. Riley EP, Infante MA, Warren KR. Fetal alcohol spectrum disorders: an overview. Neurophychol Rev (2011) 21:73-80. doi:10.1007/s11065-011-9166-x

7. Schneider ML, Moore CF, Adkins MM. The effects of prenatal alcohol exposure on behavior: rodent and primate studies. Neuropsychol Rev (2011) 21:186-203. doi:10.1007/s11065-011-9168-8

8. Valenzuela CF, Morton RA, Diaz MR, Topper L. Does moderate drinking harm the fetal brain? Insights from animal models. Trends Neurosci (2012) 35:284-92. doi:10.1016/j.tins.2012.01.006

9. Weinberg J, Silwowska JH, Lan N, Hellemans KG. Prenatal alcohol exposure: foetal programming, the hypothalamic-pituitary-adrenal axis and sex differences in outcome. J Neuroencodrinol (2008) 20:470-88. doi:10.1111/j.13652826.2008.01669.x

10. O'Connor MJ, Shah B, Whaley S, Cronin P, Gunderson B, Graham J. Psychiatric illness in clinical sample of children with prenatal alcohol exposure. Am J Drug Alcohol Abuse (2002) 28:743-54. doi:10.1081/ADA- 120015880

11. Pei J, Denys K, Hughes J, Rasmussen C. Mental health issues in fetal alcohol spectrum disorders. J Ment Health (2011) 20:473-83. doi:10.3109/09638237. 2011.577113

12. Streissguth AP, Aase JM, Clarren SK, Randels SP, LaDue RA, Smith DF. Fetal alcohol syndrome in adolescent and adults. JAMA (1991) 265:1961-7. doi:10.1001/jama.265.15.1961

13. Streissguth AP, Bookstein FL, Barr HM, Sampson PD, O’Malley K, Young JK. Risk factors for adverse life outcomes in fetal alcohol syndrome and fetal alcohol effects. J Dev Behav Pediatr (2004) 25:228-38. doi:10.1097/00004703200408000-00002

14. Herman JP, Ostrander MM, Mueller NK, Figueiredo H. Limbic system mechanisms of stress regulation: hypothalamo-pituitary-adrenocortical axis. Prog Neuropsychopharmacol Biol Psychiatry (2005) 29:1201-13. doi:10.1016/j. pnpbp.2005.08.006

15. Myers B, McKlveen JM, Herman JP. Neural regulation of the stress response: the many faces of feedback. Cell Mol Neurobiol (2012) 32:683-94. doi:10.1007/ s10571-012-9801-y

16. Drevets WC, Price JL, Furey ML. Brain structural and functional abnormalities in mood disorders: implications for neurocircuitry models of depression. Brain Struct Funct (2008) 213:93-118. doi:10.1007/s00429-008-0189-x

17. Krishnan V, Nestler EJ. Linking molecules to mood: new insight into the biology of depression. Am J Psychiatry (2010) 167:1305-20. doi:10.1176/appi.ajp. 2009.10030434

18. Fryer SL, Tapert SF, Mattson SN, Paulus MP, Spadonim AD, Riley SP. Prenatal alcohol exposure affects frontal-striatal BOLD response during inhibitory control. Alcohol Clin Exp Res (2007) 31:1415-24. doi:10.1111/j.1530-0277.2007. 00443.x

19. Norman AL, Crocker N, Mattson SN, Riley EP. Neuroimaging and fetal alcohol spectrum disorders. Dev Disabil Res Rev (2009) 15:209-17. doi:10.1002/ddrr.72

20. Riikonen R, Salonen I, Partanen K, Verho S. Brain perfusion SPECT and MRI in foetal alcohol syndrome. Dev Med Child Neurol (1999) 41:652-9. doi:10.1017/S0012162299001358

21. Willoughby KA, Sheard ED, Nash K, Rovet J. Effects of prenatal alcohol exposure on hippocampal volume, verbal learning, and verbal and spatial recall in late childhood. J Int Neuopsychol Soc (2008) 14:1022-33. doi:10.1017/ S1355617708081368

22. Haley DW, Handmaker NS, Lowe J. Infant stress reactivity and prenatal alcohol exposure. Alcohol Clin Exp Res (2006) 30:2055-64. doi:10.1111/j.1530-0277. 2006.00251.x 
23. Jacobson SW, Bihun JT, Chiodo LM. Effects of prenatal alcohol and cocaine exposure on infant cortisol levels. Dev Psychopathol (1999) 11:195-208. doi:10.1017/S0954579499002011

24. Ramsay DS, Bendersky MI, Lewis M. Effect of prenatal alcohol and cigarette exposure on two- and six-month-old infants' adrenocortical reactivity to stress. J Pediatr Psychol (1996) 21:833-40. doi:10.1093/jpepsy/21.6.833

25. Lee S, Imaki T, Vale W, Rivier C. Effects of prenatal ethanol exposure to ethanol on the activity of the hypothalamic-pituitary-adrenal axis of the offspring: importance of the time of exposure to ethanol and possible modulating mechanisms. Mol Cell Neurosci (1990) 1:168-77. doi:10.1016/1044-7431(90)90022-V

26. Lee S, Rivier C. Gender differences in the effects of prenatal alcohol exposure on the hypothalamic-pituitary-adrenal axis response to immune signals. Psychoneuroendocrinology (1996) 21:145-55. doi:10.1016/0306-4530(95) 00038-0

27. Nelson LR, Taylor AN, Lewis JW, Poland RE, Redei E, Branch BJ. Pituitaryadrenal responses to morphine and footshock stress are enhanced following prenatal alcohol exposure. Alcohol Clin Exp Res (1986) 10:397-402. doi:10.1111/j.1530-0277.1986.tb05112.x

28. Redei E, Halasz I, Li LF, Prystowsky MB, Aird F. Maternal adrenalectomy alters the immune and endocrine functions of fetal alcohol-exposed male offspring. Endocrinology (1993) 133:452-60. doi:10.1210/endo.133.2.8344191

29. Taylor AN, Branch BJ, Liu SH, Kokka N. Long-term effects of fetal ethanol exposure on pituitary-adrenal response to stress. Pharmacol Biochem Behav (1982) 16:585-9. doi:10.1016/0091-3057(82)90420-8

30. Weinberg J. Hyperresonsiveness to stress: differential effects of prenatal ethanol on males and females. Alcohol Clin Exp Res (1988) 12:647-52. doi:10.1111/j. 1530-0277.1988.tb00258.x

31. Hellemans KG, Verma P, Yoon E, Yu WK, Weinberg J. Prenatal alcohol exposure increases vulnerability to stress and anxiety-like disorders in adulthood. Ann N Y Acad Sci (2008) 1144:154-75. doi:10.1196/annals.1418.016

32. Hellemans KG, Verma P, Yoon E, Yu WK, Young AH, Weinberg J. Prenatal alcohol exposure and chronic mild stress differentially alter depressive- and anxiety-like behaviors in male and female offspring. Alcohol Clin Exp Res (2010) 34:633-45. doi:10.1111/j.1530-0277.2009.01132.x

33. O'Connor MJ, Paley B. The relationship of prenatal alcohol exposure and postnatal environment to child depressive symptoms. J Pediatr Psychol (2006) 31:50-64. doi:10.1093/jpepsy/jsj021

34. Duncan GE, Knapp DJ, Breese GR. Neuroanatomical characterization of Fos induction in rat behavior models of anxiety. Brain Res (1996) 713:79-91. doi:10.1016/0006-8993(95)01486-1

35. File SE, Zangrossi H Jr, Sanders FL, Mabbutt PS. Raised corticosterone in the rat after exposure to the elevated plus-maze. Psychopharmacology (1994) 113:543-6. doi:10.1007/BF02245237

36. Hunter MA, Takane Y. Constrained principal component analysis: various application. J Educ Behav Stat (2002) 22:105-45. doi:10.3102/ 10769986027002105

37. Hyman JM, Whitman J, Emberly E, Woodward TS, Seamans JK. Action and outcome activity state patterns in the anterior cingulate cortex. Cereb Cortex (2013) 23:1257-68. doi:10.1093/cercor/bhs104

38. Lavigne KM, Hofman S, Ring AJ, Ryder AG, Woodward TS. The personality of meaning in life: associations between dimensions of life meaning and the big five. J Posit Psychol (2013) 8:34-43. doi:10.1080/17439760.2012.736527

39. Metzak P, Feredoes E, Takane Y, Wang L, Weinstein S, Cairo T, et al. Constrained principal component analysis reveals functionally connected load-dependent networks involved in multiple stages of working memory. Hum Brain Mapp (2011) 32:856-71. doi:10.1002/hbm.21072

40. Takane Y, Shibayama T. Principal component analysis with external information on both subjects and variables. Psychometrika (1991) 56:97-120. doi:10.1007/BF02294589

41. Woodward TS, Feredoes E, Metzak PD, Takane Y, Manoach DS. Epochspecific functional networks involved in working memory. Neuroimage (2013) 65:529-39. doi:10.1016/j.neuroimage.2012.09.070

42. Lan N, Yamashita F, Halpert AG, Ellis L, Yu WK, Viau V, et al. Prenatal ethanol exposure alters the effects of gonadectomy on hypothalamicpituitary-adrenal activity in male rats. J Neuroendocrinol (2006) 18:672-84. doi:10.1111/j.1365-2826.2006.01462.x

43. Weinberg J. Effects of ethanol and maternal nutritional status on fetal development. Alcohol Clin Exp Res (1985) 9:49-55. doi:10.1111/j.1530-0277.1985. tb05049.x
44. Gallo PV, Weinberg J. Corticosterone rhythmicity in the rat: interactive effects of dietary restriction and schedule of feeding. J Nutr (1981) 111:208-18.

45. Krieger DT. Food and water restriction shifts corticosterone, temperature, activity, and brain amine periodically. Endocrinology (1974) 95:1195-202. doi:10.1210/endo-95-5-1195

46. Paxinos G, Watson C. The Rat Brain in Stereotaxic Coordinates. San Diego: Academic Press (2005).

47. Cattell RB. The scree test for the number of factors. Multivar Behav Res (1966) 1:245-76. doi:10.1207/s15327906mbr0102_10

48. Cattell RB, Vogelmann S. A comprehensive trial of the scree and KG criteria for determining the number of factors. Multivar Behav Res (1966) 12:289-325. doi:10.1207/s15327906mbr1203_2

49. Takane Y. Constrained Principal Component Analysis and Related Techniques. Boca Raton: CRC Press (2013).

50. Takane Y, Hunter MA. Constrained principal component analysis: a comprehensive theory. Appl Algebr Eng Comm (2001) 12:391-419. doi:10.1073/pnas. 1306309110

51. Mairesse J, Viltart O, Salomé N, Giuliani A, Catalani A, Casolini P, et al. Prenatal stress alters the negative correlation between neuronal activation in limbic regions and behavioral responses in rats exposed to high and low anxiogenic environments. Psychoneuroendocrinoloigy (2007) 32:765-76. doi:10.1016/j.psyneuen.2007.03.013

52. Nestler EJ, Barrot M, DiLeone RJ, Eisch AJ, Gold SJ, Monteggia LM. Neurobiology of depression. Neuron (2002) 34:13-25. doi:10.1016/S0896-6273(02) 00653-0

53. Ressler KJ, Mayberg HS. Targeting abnormal neural circuits in mood and anxiety disorders: from the laboratory to the clinic. Nat Neurosci (2007) 10:1116-24. doi:10.1038/nn1944

54. Raineki C, Cortés MR, Belnoue L, Sullivan RM. Effects of early-life abuse differ across development: infant social behavior deficits are followed by adolescent depressive-like behaviors mediated by the amygdala. J Neurosci (2012) 32:7758-65. doi:10.1523/JNEUROSCI.5843-11.2012

55. Shekhar A, Truitt W, Rainnie D, Sajdyk T. Role of stress, corticotrophin releasing factor (CRF) and amygdala plasticity in chronic anxiety. Stress (2005) 8:209-19. doi:10.1080/10253890500504557

56. Coryell MW, Wunsch AM, Haenfler JM, Allen JE, Schinzler M, Ziemann AE, et al. Acid-sensing ion channel-1a in the amygdala, a novel therapeutic target in depression related behavior. J Neurosci (2009) 29:5381-8. doi:10.1523/ JNEUROSCI.0360-09.2009

57. Duncan GE, Breese GR, Criswell H, Stumpf WE, Mueller RE, Cavey JB. Effects of antidepressant drugs injected into the amygdala on behavioral responses of rats in the forced swim test. J Pharmacol Exp Ther (1986) 238:758-62.

58. de Souza EB, Perrin MH, Insel TR, Rivier J, Vale WW, Kuhar MJ. Corticotropinreleasing factor receptors in rat forebrain: autoradiographic identification. Science (1984) 224:1449-51. doi:10.1126/science.6328656

59. Sakanaka M, Shibasaki T, Lederis K. Distribution and efferent projections of corticotropin-releasing factor-like immunoreactivity in the rat amygdaloid complex. Brain Res (1986) 382:213-38. doi:10.1016/0006-8993(86)91332-6

60. Rainnie DG, Bergeron R, Sajdky TJ, Patil M, Gehlert DR, Shekhar A. Corticotrophin releasing factor-induced synaptic plasticity in the amygdala translates stress into emotional disorders. J Neurosci (2004) 24:3471-9. doi:10.1523/ JNEUROSCI.5740-03.2004

61. Sajdyk TJ, Schober DA, Gehlert DR, Shekhar A. Role of corticotropin-releasing factor and urocortin within the basolateral amygdala of rats in anxiety and panic responses. Behav Brain Res (1999) 100:207-15. doi:10.1016/S01664328(98)00132-6

62. Ulrich-Lai YM, Herman JP. Neural regulation of endocrine and autonomic stress response. Nat Rev Neurosci (2009) 10:397-409. doi:10.1038/nrn2647

63. Fanselow MS, Dong HW. Are the dorsal and ventral hippocampus functionally distinct structures? Neuron (2010) 65:7-19. doi:10.1016/j.neuron. 2009.11.031

64. Bannerman DM, Rawlins JN, McHugh SB, Deacon RM, Yee BK, Bast T, et al. Regional dissociations within the hippocampus-memory and anxiety. Neurosci Biobehav Rev (2004) 28:273-83. doi:10.1016/j.neubiorev.2004.03.004

65. Adhikari A, Topiwala MA, Gordon JA. Synchronized activity between the ventral hippocampus and medial prefrontal cortex during anxiety. Neuron (2010) 65:257-69. doi:10.1016/j.neuron.2009.12.002

66. Adhikari A, Topiwala MA, Gordon JA. Single units in the medial prefrontal cortex with anxiety-related firing patterns are preferentially influenced by ventral 
hippocampus activity. Neuron (2011) 71:898-910. doi:10.1016/j.neuron.2011. 07.027

67. Herman JP, Figueiredo H, Mueller NK, Ulrich-Lai Y, Ostrander MM, Choi DC, et al. Central mechanisms of stress integration: hierarchical circuitry controlling hypothalamo-pituitary-adrenocortical responsiveness. Front Neuroendocrinol (2003) 24:151-80. doi:10.1016/j.yfrne.2003.07.001

68. Dunn JD, Orr SE. Differential plasma corticosterone responses to hippocampal stimulation. Exp Brain Res (1984) 54:1-6. doi:10.1007/BF00235813

69. West JR, Hodges CA, Black AC. Prenatal exposure to ethanol alters the organization of hippocampal mossy fibres in rats. Science (1981) 211:957-9. doi:10.1126/science.7466371

70. Abel EL, Jacobson S, Sherwin BT. In utero alcohol exposure: functional and structural brain damage. Neurobehav Toxicol Teratol (1983) 5:363-6.

71. Christie BR, Swann SE, Fox CJ, Froc D, Lieblich SE, Redila C, et al. Voluntary exercise rescues deficits in spatial memory and long-term potentiation in prenatal ethanol-exposed male rats. Eur J Neurosci (2005) 21:1719-26. doi:10.1111/j.1460-9568.2005.04004.x

72. Sutherland RJ, McDonald RJ, Savage DD. Prenatal exposure to moderate levels of ethanol can have long-lasting effects on hippocampal synaptic plasticity in adult offspring. Hippocampus (1997) 7:232-8. doi:10.1002/(SICI)10981063(1997) 7:2<232::AID-HIPO9>3.3.CO;2-O

73. Redila VA, Olson AK, Swann SE, Mohades G, Webber AJ, Weinberg J, et al. Hippocampal cell proliferation is reduced following prenatal ethanol exposure but can be rescued with voluntary exercise. Hippocampus (2006) 16:305-11. doi:10.1002/hipo.20164

74. Sliwowska JH, Barker JM, Barha CK, Lan N, Weinberg J, Galea LA. Stressinduced suppression of hippocampal neurogenesis in adult male rats is altered by prenatal ethanol exposure. Stress (2010) 13:302-14. doi:10.3109/ 10253890903531582

75. Glavas MM, Ellis L, Yu WK, Weinberg J. Effects of prenatal ethanol exposure on basal limbic-hypothalamic-pituitary-adrenal regulation: role of corticosterone. Alcohol Clin Exp Res (2007) 31:1598-610. doi:10.1111/j.1530-0277. 2007.00460.x

76. Kim CK, Yu W, Edin G, Ellis L, Osborn JA, Weinberg J. Chronic intermittent stress does not differentially alter brain corticosteroid receptor densities in rats prenatally exposed to ethanol. Psychoneuroendocrinology (1999) 24:585-611. doi:10.1016/S0306-4530(99)00015-3

77. Uban KA, Comeau WL, Ellis LA, Galea LA, Weinberg J. Basal regulation of HPA and dopamine systems is altered differentially in males and females by prenatal alcohol exposure and chronic variable stress. Psychoneuroendocrinology (2013) 38:1953-66. doi:10.1016/j.psyneuen.2013.02.017

78. Jayatissa MN, Bisgaard CF, West MJ, Wiborg O. The number of granule cells in rat hippocampus is reduced after chronic mild stress and re-established after chronic escitalopran treatment. Neuropharmacology (2008) 54:530-41. doi:10.1016/j.neuropharm.2007.11.009

79. Lee KL, Kim SJ, Kim SJ, Choi SH, Shin YC, Park SH, et al. Chronic mild stress decreases survival, but nor proliferation, of new-born cells in adult rat hippocampus. Exp Mol Med (2006) 38:44-54. doi:10.1038/emm.2006.6

80. Lacroix L, Spinelli S, Heidbreader CA, Feldon J. Differential role of the medial and lateral prefrontal cortices in fear and anxiety. Behav Neurosci (2000) 114:1119-30. doi:10.1037/0735-7044.114.6.1119

81. Resstel LB, Souza RF, Guimarães FS. Anxiolytic-like effects induced by medial prefrontal cortex inhibition in rats submitted to the Vogel conflict test. Physiol Behav (2008) 93:200-5. doi:10.1016/j.physbeh.2007.08.009

82. Shah AA, Treit D. Excitotoxic lesions of the medial prefrontal cortex attenuates fear responses in the elevated-plus maze, social interaction and shock probe burying tests. Brain Res (2003) 969:183-94. doi:10.1016/S0006-8993(03) 02299-6

83. Jinks AL, McGregor IS. Modulation of anxiety-related behaviours following lesions of the prelimbic or infralimbic cortex in the rat. Brain Res (2007) 772:181-90. doi:10.1016/S0006-8993(97)00810-X

84. de Visser L, Baars AM, van't Klooster J, van den Bos R. Transient inactivation of the medial prefrontal cortex affects both anxiety and decision-making in male Wistar rats. Front Neurosci (2011) 5:102. doi:10.3389/fnins.2011.00102

85. Figueiredo HF, Bruestle A, Bodie B, Doglas CM, Herman JP. The medial prefrontal cortex differentially regulates stress induced $c$-fos expression in the forebrain depending on type of stressor. Eur J Neurosci (2003) 18:2357-64. doi:10.1046/j.1460-9568.2003.02932.x
86. Radley JJ, Arias CM, Sawchenko PE. Regional differentiation of the medial prefrontal cortex in regulating adaptive responses to acute emotional stress. J Neurosci (2006) 26:12967-76. doi:10.1523/JNEUROSCI.4297-06.2006

87. Radley JJ, Gosselink KL, Sawchenko PE. A discrete GABAergic relay mediated medial prefrontal cortical inhibition of the neuroendocrine stress response. J Neurosci (2009) 29:7330-40. doi:10.1523/JNEUROSCI.5924-08.2009

88. Charmandari E, Tsigos C, Chrousos G. Endocrinology of the stress response. Annu Rev Physiol (2005) 67:259-84. doi:10.1146/annurev.physiol.67.040403. 120816

89. Lee S, Schmidt D, Tilders F, Rivier C. Increased activity of the hypothalamicpituitary-adrenal axis of rats exposed to alcohol in utero: role of altered pituitary and hypothalamic function. Mol Cell Neurosci (2000) 16:515-28. doi: $10.1006 /$ mcne. 2000.0890

90. Bondi CO, Rodriguez G, Gould GG, Frazer A, Morilak DA. Chronic unpredictable stress induces cognitive deficit and anxiety-like behavior in rats that is prevented by chronic antidepressant drug treatment. Neuropsychopharmacology (2008) 33:320-31. doi:10.1038/sj.npp.1301410

91. Christiansen S, Bouzinova EV, Palme R, Wiborg O. Circadian activity of the hypothalamic-pituitary-adrenal axis is differentially affected in the rat chronic mild stress model of depression. Stress (2012) 15:647-57. doi:10.3109/ 10253890.2011.654370

92. Hill MN, Hellemans KG, Verma P, Gorzalka BB, Weinberg J. Neurobiology of chronic mild stress: parallels to major depression. Neurosci Biobehav Rev (2012) 36:2085-117. doi:10.1016/j.neubiorev.2012.07.001

93. Willner P, Towell A, Sampson D, Sophokleous S, Muscat R. Reduction of sucrose preference by chronic unpredictable mild stress, and its restoration by tricyclic antidepressant. Psychopharmacology (1987) 93:358-64. doi:10.1007/ BF00187257

94. Michelsen KA, van der Hove DL, Schmitz C, Segers O, Prickaerts J, Steinbusch HW. Prenatal stress and subsequent exposure to chronic mild stress influence dendritic spine density and morphology in the rat medial prefrontal cortex. BMC Neurosci (2007) 8:107. doi:10.1186/1471-2202-8-107

95. Radley JJ, Rocher AB, Janssen WG, Hof PR, McEwen BC, Morrison JH. Reversibility of apical dendritic retraction in the rat medial prefrontal cortex following repeated stress. Exp Neurol (2005) 196:199-203. doi:10.1016/j. expneurol.2005.07.008

96. Radley JJ, Rocher AB, Rodriguez A, Ehlenberger DB, Dammann M, McEwen BS, et al. Repeated stress alters dendritic spine morphology in the rat medial prefrontal cortex. J Comp Neurol (2008) 507:1141-50. doi:10.1002/cne.21588

97. Banasr M, Duman RS. Glial loss in the prefrontal cortex is sufficient to induce depressive-like behaviors. Biol Psychiatry (2008) 64:863-70. doi:10. 1016/j.biopsych.2008.06.008

98. Arnsten AF. Stress signalling pathways that impair prefrontal cortex structure and function. Nat Rev Neurosci (2009) 10:410-22. doi:10.1038/nrn2648

99. Liston C, Miller MM, Goldwater DS, Radley JJ, Rocher AB, Hof PR, et al. Stress-induced alterations in prefrontal cortical dendritic morphology predict selective impairments in perceptual attentional set-shifting. J Neurosci (2006) 26:7870-4. doi:10.1523/JNEUROSCI.1184-06.2006

Conflict of Interest Statement: The authors declare that the research was conducted in the absence of any commercial or financial relationships that could be construed as a potential conflict of interest.

Received: 31 October 2013; accepted: 10 January 2014; published online: 13 February 2014.

Citation: Raineki C, Hellemans KGC, Bodnar T, Lavigne KM, Ellis L, Woodward TS and Weinberg $J$ (2014) Neurocircuitry underlying stress and emotional regulation in animals prenatally exposed to alcohol and subjected to chronic mild stress in adulthood. Front. Endocrinol. 5:5. doi: 10.3389/fendo.2014.00005

This article was submitted to Neuroendocrine Science, a section of the journal Frontiers in Endocrinology.

Copyright (c) 2014 Raineki, Hellemans, Bodnar, Lavigne, Ellis, Woodward and Weinberg. This is an open-access article distributed under the terms of the Creative Commons Attribution License (CC BY). The use, distribution or reproduction in other forums is permitted, provided the original author(s) or licensor are credited and that the original publication in this journal is cited, in accordance with accepted academic practice. No use, distribution or reproduction is permitted which does not comply with these terms. 